\title{
Valores, atitudes e comportamentos ambientais em estudantes do Ensino Médio em uma Escola Pública na Amazônia
}

\author{
Environmental values, attitudes and behaviors in high school students in a public School \\ in the Amazonia/Brazil
}

\section{Valores, actitudes y comportamientos ambientales en estudiantes de secundaria de una escuela pública de la Amazonía/Brazil}

\author{
Jonas da Paz Aguiar ${ }^{1}$ \\ Cláudia Silva de Castro ${ }^{2}$ \\ Asdrubal Jesus Farias-Ramirez ${ }^{3}$
}

\begin{abstract}
Resumo
A Educação Ambiental tem sido discutida como das principais estratégias diante dos impactos ambientais crescentes, provocados pelas atividades humanas. No Brasil, apesar das importantes conquistas legais quanto à Educação Ambiental, na prática, a sua implementação na Educação Básica tem enfrentado grandes dificuldades. Entre outros motivos está a ausência da utilização de mecanismos avaliativos que permitam avaliações diagnósticas e o planejamento estratégico para a Educação Ambiental mais prática e eficiente. Nesse contexto, o objetivo deste trabalho foi aplicar um modelo amplo de diagnóstico quanto aos valores, atitudes e comportamentos ambientais de estudantes do Ensino Médio, analisando as diferenças entre sexo, nível de escolaridade e idade e correlações entre as variáveis ambientais. Foi aplicado um questionário com escalas de valores, atitudes e comportamentos ambientais para uma amostra de 138 estudantes, de ambos os sexos, matriculados no primeiro, segundo e terceiro anos do Ensino Médio. Foi encontrada uma maior concordância das respostas dos estudantes com os valores ecocêntricos, seguido dos valores antropocêntricos. Estudantes do sexo feminino foram mais concordantes com os valores antropocêntricos e estudantes do primeiro ano foram mais apáticos às questões ambientais quando comparados com os alunos do terceiro ano do Ensino Médio. Também, foram encontradas correlações entre as respostas aos valores ecocêntricos e atitudes ambientais, valores ecocêntricos e comportamentos ambientais, valores antropocêntricos e apáticos, atitudes e comportamentos ambientais. A aplicação das escalas forneceu informações relevantes que podem ser consideradas no planejamento de práticas de Educação Ambiental, indicando pontos da relação ambiental que podem ser prioritários.
\end{abstract}

Palavras-chave: Educação Ambiental. Escalas. Antropocêntricos. Ecocêntricos.

\section{Resumen}

La Educación Ambiental ha sido tratada como una de las principales estrategias ante los crecientes impactos ambientales provocados por las actividades humanas. En Brasil, a pesar de los importantes logros legales en materia de Educación Ambiental, en la práctica, en la Educación Básica su implementación ha enfrentado grandes dificultades. Entre otras razones, está la ausencia del uso de mecanismos de evaluación que permitan realizar diagnósticos para una planificación estratégica de la Educación Ambiental más práctica y eficiente. En este contexto, el objetivo de este trabajo fue aplicar un modelo de diagnóstico amplio sobre los valores, actitudes y comportamientos ambientales de los estudiantes de secundaria; analizando las diferencias entre sexo, nivel educativo y edad; y las correlaciones entre las variables ambientales. Un cuestionario con escalas de valores, actitudes y comportamientos ambientales se aplicó a una muestra de 138 estudiantes, de ambos os sexos, de los primeros, segundos y terceros años de escuela pública secundaria. Se encontró una mayor concordancia entre las respuestas de los estudiantes y los valores ecocéntricos, seguidos de los valores antropocéntricos. Las alumnas estaban más de acuerdo con los valores antropocéntricos, y los estudiantes de primer año fueron más apáticos hacia los problemas ambientales en comparación con los estudiantes de tercer año de secundaria. También fueron

\footnotetext{
${ }^{1}$ Doutor em Ciências Ambientais pela Universidade Federal do Pará. Docente da Rede do Estado Pará. E-mail: jonaspaguiar@gmail.com

${ }^{2}$ Doutora em Educação em Ciências e Matemática pela Universidade Federal de Mato Grosso. Docente da Universidade Federal do Oeste do Pará. Email: claus.castro@ hotmail.com

${ }^{3}$ Doutor em Engenharia de Sistemas pela Universidade de São Paulo. Faculdade Pecege, Programa de Educação Continuada em Economia e Gestão de Empresas (PECEGE). E-mail: ajfara@gmail.com
} 
encontradas correlaciones entre las respuestas a los valores ecocéntricos y las actitudes ambientales; los valores ecocéntricos y los comportamientos ambientales; los valores antropocéntricos y los apáticos; las actitudes y los comportamientos ambientales. La aplicación de las escalas proporcionó información relevante que puede ser considerada al momento de planificar las actividades de Educación Ambiental, indicando los puntos que pueden ser prioritarios dentro del tema ambiental.

Palabras clave: Educación Ambiental. Escalas. Antropocéntrico. Ecocéntrico.

\begin{abstract}
Environmental Education has been considered as one of the main strategies in view of the increased environmental impacts, produced by human activities. Despite, the important legal achievements regarding Environmental Education in Brazil, in practice, its implementation in Basic Education has faced great difficulties. Among other reasons, the absence of the use of evaluation mechanisms that allow diagnostic evaluations and strategic planning for more practical and efficient Environmental Education. In this context, the aim of this study was to apply a broad model of diagnosis and the values, attitudes and environmental behavior of high school students by analyzing the differences between sex, education level and age and correlations between environmental variables. A questionnaire with scales of values, attitudes and environmental behaviors was applied to a sample of 138 students from the first, second and third high schools of both sexes. A greater agreement was found between the students' responses and ecocentric values, followed by anthropocentric values. Female students were more in agreement with anthropocentric values and first-year students were more apathetic to environmental issues when compared to third-year high school students. Correlations were also found between responses to ecocentric values and environmental attitudes, ecocentric values and environmental behaviors, anthropocentric values and apathetic environmental attitudes and behaviors. The application of the scales provided relevant information that can be considered when planning Environmental Education practices, indicating points of the environmental issue that may be a priority.
\end{abstract}

Keywords: Environmental Education. Scale. Anthropocentric. Ecocentric.

\title{
1 Introdução
}

O aquecimento global, derretimento das calotas polares, extinção de espécies, desertificação de áreas, poluição dos recursos hídricos e esgotamento dos recursos naturais são alguns dos problemas ambientais que têm despertado preocupações quanto ao futuro da vida na terra (SAKA; SURMELI, 2013; DESJARDINS, 2013). Os primeiros movimentos enfáticos, frente a essas preocupações, surgiram na década 1960, motivados, principalmente, pelo livro Primavera Silenciosa, escrito por Rachel Carson em 1962, sobre os efeitos deletérios do uso de pesticida, e pela criação do Grupo de Roma, em 1968 (CULVER; MAUCH; RITSON, 2012; NOVO, 2014; MIHAILOV; SAKELARIEVA, 2016).

Nas décadas posteriores, motivadas pelas preocupações ambientais crescentes, as discussões sobre a necessidade da implementação de políticas de Educação Ambiental se intensificaram e foram temas centrais de conferências internacionais, como na Reunião Internacional de Trabalhos sobre Educação Ambiental no Currículo Escolar, promovida IUCN/UNESCO, em 1970, Nevada - EUA; na Assembleia das Nações Unidas, em 1972; no Workshop Internacional sobre Educação Ambiental, em 1975, realizado pela UNESCO/ PNUMA, em Belgrado; na Conferência Intergovernamental de Educação Ambiental, em 1977, Tbilisi - Geórgia; no Congresso Internacional sobre Educação e Treinamento Ambiental, em 1987, em Moscou; na Conferência das Nações Unidas sobre o Meio Ambiente e o Desenvolvimento, em 1992, Rio de Janeiro - Brasil, também chamada de Rio - 92 e nas conferências que seguiram-na, Rio+10 e Rio+20 (AMEMIYA; MACER, 1999; DAS, 2001; KOPNINA, 2012).

No Brasil, os movimentos para programas de Educação Ambiental tiveram início ainda na década de 1970, através de várias manifestações: ações de organizações da sociedade civil, 
de prefeituras e de governos estaduais, influenciados, principalmente, pelas iniciativas internacionais (DIAS, 1991). De forma institucionalizada, somente a partir de 1981, com a publicação da Lei $n^{\circ}$ 6.938, que dispunha sobre a Política Nacional do Meio Ambiente e, mais tarde, com a Constituição Federal do Brasil, promulgada no ano de 1988. Avanços importantes na Educação Ambiental foram obtidos com Lei de Diretrizes e Base da Educação, Lei de N $^{\circ}$ 9394, de 1996, e com a Política Nacional de Educação Ambiental, Lei № 9795, de 1999 (MARCATTO, 2002).

A Política Nacional de Educação Ambiental (BRASIL, 1999) foi um marco importante para as práticas de Educação Ambiental, estabelecendo, por exemplo, que a mesma deve estar presente em todos os níveis e modalidades de ensino, sendo um elemento essencial e permanente da educação nacional, em caráter formal e não formal.

$\mathrm{Na}$ Amazônia, os projetos de Educação Ambiental emergem em um cenário marcado por conflitos diante de distintos interesses político-econômicos e de identidade diversificada, com iniciativas de projetos de Educação Ambiental, frequentemente desenvolvidos de forma isolada (ANDRADE; GÓMEZ, 2017)

Apesar dos grandes avanços legislativos, na prática, a sua implementação na Educação Básica tem enfrentado grandes dificuldades (NUNES; FRANÇA; PAIVA, 2017; MACHADO; TERÉN, 2018). Entre as dificuldades, está a necessidade de se adotarem ferramentas amplas de avaliação diagnóstica, incluindo aspectos de predisposição ambientais, como valores relacionados à ética ambiental, atitudes e comportamentos, que possam fornecer informações relevantes para o planejamento de estratégias de Educação Ambiental, de modo mais prático e eficiente.

Embora escalas de avaliação, relacionadas à educação ambiental, venham sendo desenvolvidas em todo mundo, geralmente, elas têm sido aplicadas de forma isolada com abordagens ora relacionadas à ética ambiental (THOMPSON; BARTON, 1994; AMEMIYA; MACER, 1999; SAKA; SURMELI, 2013), ora abordando as atitudes e os comportamentos ambientais (HEYL; DÍAS; CIFUENTES, 2014; BOCA; SARAÇLI, 2019).

Heyl, Díaz e Cifuentes (2014) conceituaram atitudes ambientais como uma predisposição para responder de maneira favorável com relação ao meio ambiente, e o comportamento ambiental como ações que ajudam na preservação e conservação ambiental.

Thompson e Barton (1994) elaboraram uma escala para ética ambiental, baseada em três subescalas: i) antropocêntricas, para mensurar a apreciação pelo bem ambiental, porém voltado para atender às necessidades humanas, ou seja, centrada no homem (SAKA; SURMELI, 2013); ii) ecocêntricas, para medir o interesse pela natureza como valor intrínseco, o que justifica a necessidade de protegê-la; iii) apáticas, destinadas para aferir a falta de interesse e descrença quanto à realidade das questões ambientais (KOPNINA, 2012).

As escalas de valores ou crenças ambientais são baseadas na ideia de que as atitudes e o comportamento ambiental dos indivíduos são influenciados por suas crenças ambientais. Dessa forma, a Educação Ambiental tem um papel fundamental na formação de um novo pensamento com valores pró-ambiental, e soluções ambientais passam por mudanças na percepção do meio ambiente (MOYANO-DÍAZ; CORNEJO; GALLARDO, 2011).

Em várias partes do mundo, escalas de atitude ambientais e comportamentos ambientais foram desenvolvidas e aplicadas (HEYL; DÍAS; CIFUENTES, 2014; BOCA; SARAÇLI, 2019).

A relação entre sexo, idade, escolaridade, nacionalidade, formação e classe social, valores, atitudes e comportamento têm sido algumas das variáveis analisadas para explicar a relação dos indivíduos com o meio ambiente (ERTEN; AYDOĞDU, 2011; SAKA; SURMELI, 2013; HEYL; DÍAZ; CIFUENTES, 2014). Para Vicente-Molina, Fernández-Sáinz e IzagirreOlaizola (2013) a Educação é um dos principais fatores que explica o comportamento ambiental, sendo que os indivíduos mais instruídos são mais preocupados e motivados com a 
questão ambiental. Diferenças quanto aos valores e comportamento ambiental entre os sexos, nacionalidade e correlação entre atitudes ambientais e comportamentos também têm sido observadas (ERTEN; AYDOĞDU, 2011; CÔRTES et al., 2016; CASALÓ; ESCARIO, 2017).

Dentro desse contexto, a aplicação de escalas ambientais em estudantes do Ensino Médio pode fornecer informações importantes para a elaboração de estratégias de Educação Ambiental, compatíveis com a realidade vivenciada nesse contexto escolar. Esta pesquisa tem como objetivo aplicar uma avaliação diagnóstica agregada de diferentes escalas relacionadas aos valores, às atitudes e aos comportamentos ambientais em estudantes do Ensino Médio, além de avaliar as diferenças e as correlações entre esses aspectos.

\section{Material e Métodos}

O presente estudo foi realizado com 138 estudantes ( 84 do sexo feminino e 54 do sexo masculino), com idade entre 14 e 19 anos, de uma escola pública localizada na região rural da cidade Santarém, Estado do Pará, Brasil. A escola referida atende alunos de mais de vinte comunidades rurais e estudantes de regiões urbanas. O estudo foi realizado com alunos dos três níveis do Ensino Médio, sendo 46 do primeiro ano, 50 do segundo ano e 42 alunos do terceiro ano, totalizando cerca de $33 \%$ dos estudantes da escola. Este trabalho foi aprovado pelo CEPESALQ/USP com parecer de número 4.145.099.

Para isso, foi adotado um amplo questionário, com 62 questões relacionadas à identificação (3-idade, nível de ensino e sexo) e a três fatores ambientais, os quais são valores (24), atitudes (20) e comportamento (15). Para todas as questões relacionadas aos valores, atitudes e comportamento ambiental, foi utilizada a escala Likert com cinco graus de conformidade: concordo totalmente, concordo parcialmente, neutro, discordo parcialmente e discordo totalmente (LIKERT, 1932).

\subsection{Escalas Ambientais}

As questões quanto aos valores ambientais foram adaptadas das desenvolvidas por Thompson e Barton (1994) e são compostas de três subescalas para medir valores ecocêntricos, antropocêntricos e apáticos em relação às questões ambientais (conforme Quadro 1). A escala de atitudes ambientais foi adaptada, utilizando questões de quatro categorias de atitudes: hábitos, voluntariado, advertência e participação, com conteúdo relacionado à energia, à água, ao transporte, à reciclagem e ao consumo (HEYL; DÍAZ; CIFUENTES, 2014; BOCA; SARAÇLI, 2019). A escala de comportamento ambiental também foi adaptada, utilizando os mesmos conteúdos adotados para a escala de atitude ambiental (PATO; ROS; TAMAYO, 2005; MOYANO-DÍAZ; ENCINA; VICENTE, 2007; HEYL; DÍAZ; CIFUENTES, 2014).

\begin{tabular}{|c|c|c|c|}
\hline Escalas & Subescalas & Questões & Adaptado de: \\
\hline \multirow[t]{3}{*}{ Valores } & Antropocêntricos (A) & 8 & \multirow{3}{*}{ Thompson e Barton (1994) } \\
\hline & Ecocêntricos $(\mathrm{E})$ & 8 & \\
\hline & Apáticos (P) & 8 & \\
\hline \multirow[t]{4}{*}{ Atitudes (At) } & Hábitos & 5 & \multirow{4}{*}{$\begin{array}{l}\text { Heyl, Díaz e Cifuentes (2014); } \\
\text { Boca e Saraçli (2019) }\end{array}$} \\
\hline & Voluntariado & 5 & \\
\hline & Advertência & 5 & \\
\hline & Participação & 5 & \\
\hline Comportamento (Co) & & 15 & $\begin{array}{l}\text { Pato, Ros e Tamayo, 2005., } \\
\text { (2005); Heyl, Díaz e Cifuentes } \\
\text { (2014); Moyano-Díaz, Encina e } \\
\text { Vicente (2007) }\end{array}$ \\
\hline
\end{tabular}

Quadro 1 - Escalas e subescalas de valores, atitudes e comportamento Fonte: Elaborado pelos autores 


\section{Análise dos dados}

Os dados dos questionários foram tabulados e processados, utilizando-se o programa da Microsoft Excel versão 16.28. A Análise de confiabilidade do questionário foi aplicada, utilizando-se o coeficiente alfa de Cronbach, sendo encontrado um valor considerado alto de 0,895 (LANDIS; KOCH, 1977). Para as análises descritivas das questões dos fatores ambientais foram calculadas a frequência absoluta e frequência relativa de cada uma das cinco respostas possíveis para a escala Likert. Também, foi calculada a moda do conjunto de dados. Todas essas análises foram feitas utilizando o software IBM SPSS Statistics 20 (KIRKPATRICK; FEENEY, 2013). A representação gráfica dos itens dos resultados foi construída, utilizando-se o pacote Likert no software $R$ (R CORE TEAM, 2020).

As diferenças entre os sexos, níveis de escolaridade e idade foram comparadas pelos testes não paramétricos de Wilcoxon e Kruskal-Wallis (KRUSKAL; WALLIS, 1952) em razão da ausência de distribuição normal na amostragem, encontrada através do teste de normalidade de Shapiro-Wilk. Essa análise utilizou-se do software IBM SPSS Statistics 20 (KIRKPATRICK; FEENEY, 2013). As hipóteses analisadas podem ser vistas no Quadro 2.

\begin{tabular}{|l|l|}
\hline Número & \multicolumn{1}{c|}{ Hipóteses } \\
\hline H1 & Valores antropocêntricos dos estudantes têm relação com valores apáticos \\
H2 & Valores antropocêntricos dos estudantes têm relação com as atitudes ambientais \\
H3 & Valores apáticos dos estudantes têm relação com as atitudes ambientais \\
H4 & Valores ecocêntricos dos estudantes têm relação com as atitudes ambientais \\
H5 & Valores antropocêntricos dos estudantes têm relação com o comportamento \\
& ambiental \\
H6 & Valores apáticos dos estudantes têm relação com o comportamento ambiental \\
H7 & Atitudes ambientais dos estudantes têm relação com o comportamento ambiental \\
H8 & Valores ecocêntricos dos estudantes têm relação com o comportamento ambiental \\
H9 & Valores antropocêntricos dos estudantes têm relação com os valores ecocêntricos \\
H10 & Valores apáticos dos estudantes têm relação com os valores ecocêntricos \\
\hline \multicolumn{2}{|c}{ Quadro 2 - Hipóteses testadas entre os fatores ambientais utilizados } \\
\hline
\end{tabular}

Para análise da relação entre as variáveis ambientais (valores, atitudes e comportamentos ambientais), foi utilizado o software de modelagem de Equações Estruturais SmartPLS versão 3.3.2 (RINGLE; WENDE; BECKER, 2015). A utilização do software SmartPLS foi adotada em razão da adequação quanto ao número amostral e ausência da distribuição normal nos dados (RINGLE; BIDO; SILVA, 2014). Valores-p foram estimados por bootstrapping com 5000 repetições.

\section{Resultados e Discussão}

A frequência absoluta, frequência relativa e moda para cada uma das perguntas quanto aos fatores ambientais são apresentados no Anexo 1, e os resultados para cada um dos itens das escalas são apresentados nas Figuras 1 e 2, respectivamente, para valores ambientais, atitudes e comportamentos. 


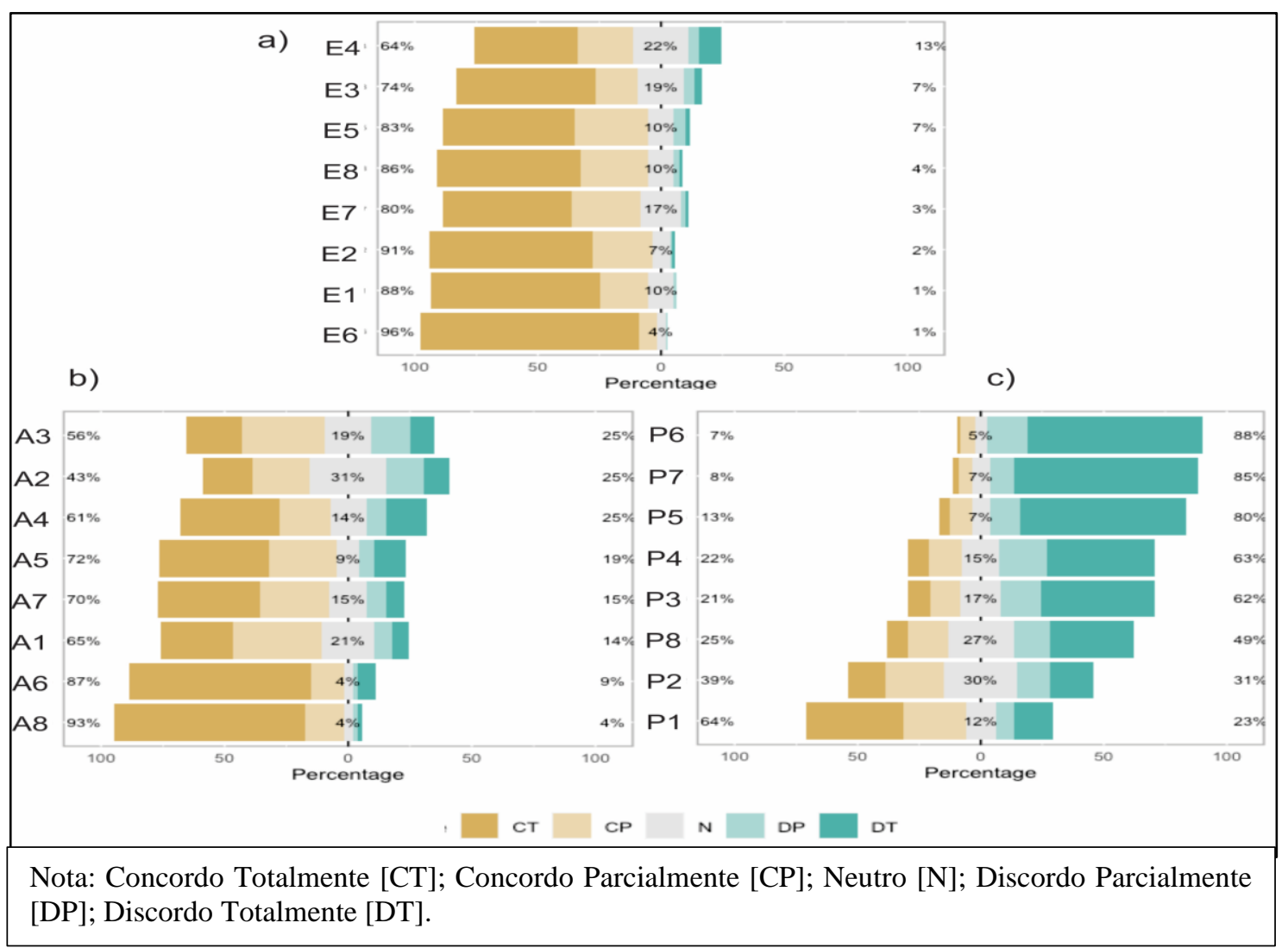

Figura 1 - Gráfico com a porcentagem para cada um dos itens da escala Likert para os valores ambientais: a) ecocêntricos (E); b) antropocêntricos (A); c) apáticos (P)

Fonte: dados da pesquisa

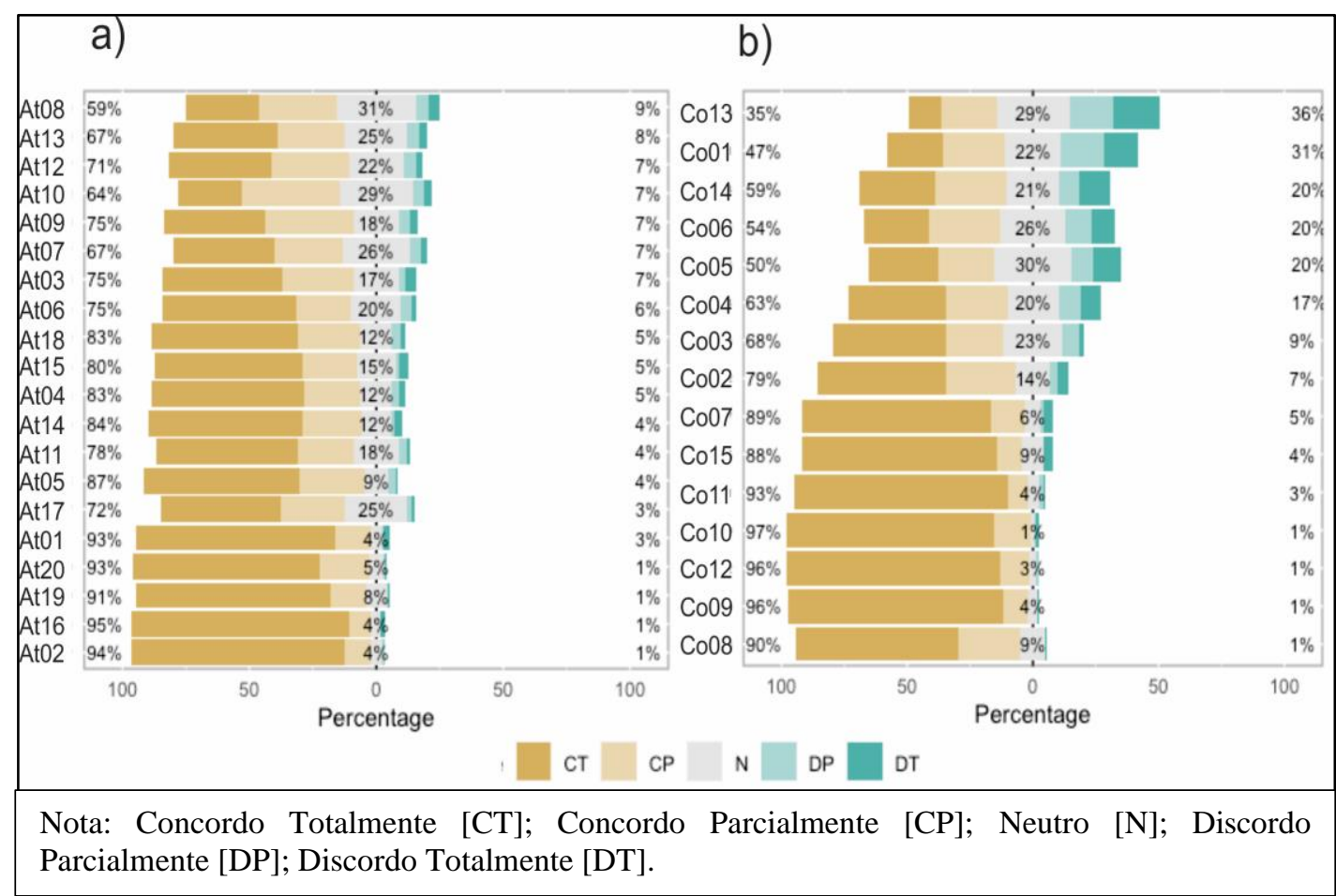

Figura 2 - Gráfico com a porcentagem para cada um dos itens da escala Likert para: a) atitudes ambientais (At); b) comportamentos ambientais (Co)

Fonte: dados da pesquisa 
De forma geral, os estudantes apresentaram maior afinidade a valores ecocêntricos $(E)$ quando comparados com os valores antropocêntricos (A) e apáticos (P) ao meio ambiente. Valores apáticos foram considerados menos aceitáveis. Resultados semelhantes foram encontrados por Erten e Aydoğdu (2011) para estudantes da Turquia e Azerbaijão, Kopnina (2017) para diferentes cursos relacionados à Gestão e a Negócios, e Paz e Higuchi (2019), para jovens integrantes de coletivos socioambientais de Manaus, Amazonas, Brasil. Nesse aspecto, os resultados encontrados são convergentes com os encontrados em outros trabalhos para uma predominância de valores ecocêntricos.

Quanto às três escalas de valores ambientais as afirmações "E6. A natureza é valiosa por si mesma" 96\%) e a "A8. A natureza é importante por que pode contribuir para o prazer e o bem-estar dos seres humanos" (93\%) foram as mais aceitáveis. As afirmações menos aceitáveis foram "P6. Eu não me importo com problemas ambientais" (5\%) e "P7. Eu sou contra programas para preservar a natureza selvagem, reduzir a poluição e conservar recursos naturais" (7\%). Por outro lado, uma parcela de estudantes considera aceitável que "P1. As ameaças ambientais como o desmatamento e a destruição da camada de ozônio são exageradas" (64\%) e que "P2. Parece-me que a maioria dos ambientalistas é pessimista e alguns são paranoicos" $(39 \%)$.

Com relação às atitudes (At) e aos comportamentos ambientais $(\mathrm{Co})$ foram encontradas alta porcentagem de resposta concordante com as afirmações mais ecológicas, sendo a moda para a maioria das informações igual a 1 (Concordo Totalmente) (Conforme Figura 2). Dentro da escala de atitude ambiental, de forma cumulativa as afirmações relacionadas ao voluntariado (At6, At7, At8, At9 e At10) foram menos concordantes. Para o comportamento ambiental, as afirmações com relação à participação direta em campanhas de reciclagem, de campanhas que cuidam do meio ambiente e de reciclagem de papel, de vidro e de latas foram as que tiveram menor receptividade (Co1, Co13 e Co14). Vicente-Molina, Fernández-Sáinz e IzagirreOlaizola (2013), em um estudo realizado com estudantes da Espanha, Estados Unidos, México e Brasil encontraram menor taxa de participação dos estudantes brasileiros em atividades de reciclagem.

Diante disso, em relação aos valores é adequado que as práticas de Educação Ambiental voltadas à população, segundo abordagem deste trabalho, busquem desmistificar crenças prejudiciais ao meio ambiente como as consideradas aceitáveis dentro da escala de valores apáticos por uma parcela considerável de estudantes. Da mesma maneira, em relação às atitudes e aos comportamentos ambientais, é importante que os programas ambientais incentivem a participação ativa dos estudantes como, por exemplo, em campanhas de reciclagem e de cuidados com o meio ambiente, tendo em vista que as afirmações relacionadas a esse tema foram menos concordantes dentro das escalas.

Em relação à análise comparativa, foram encontradas diferenças significativas entre os resultados relacionados aos valores antropocêntricos em razão do sexo (conforme Tabela 1). As respostas do sexo feminino apresentaram maior concordância com os valores antropocêntricos. Erten e Aydoğdu (2011) também encontraram maior afinidade entre o sexo feminino e os valores antropocêntricos para estudantes da Turquia e Azerbaijão. Valores antropocêntricos são, geralmente, relacionados a pensamentos baseados em interesse pessoal (KOPNINA, 2017; DESJARDINS, 2013). Aparentemente, para o sexo feminino esses valores são mais aceitáveis. No entanto, tais resultados não foram refletidos em atitudes e em comportamentos ambientais, tendo em vista que não foram encontradas diferenças significativas para esses fatores em razão do sexo. 
Tabela 1 - Análise comparativa quanto à diferença dos resultados dos valores, atitudes e comportamentos ambientais em razão do sexo, nível de ensino e idade.

\begin{tabular}{|c|c|c|c|c|c|}
\hline Item & & Análise comparativa & Teste & Signif. & Resultados \\
\hline 1. & Sexo & $\begin{array}{l}\text { V. antropocêntricos } \\
\text { (A) } \\
\text { V. ecocêntricos (E) } \\
\text { V. apáticos (P) } \\
\text { Atitudes (At) } \\
\text { Comportamentos } \\
\text { (Co) }\end{array}$ & Wilcoxon W & $\begin{array}{l}\mathbf{0 , 0 0 *} \\
0,17 \\
0,07 \\
0,29 \\
0,89\end{array}$ & $\begin{array}{l}\text { Significante somente para } \\
\text { valores antropocêntricos. } \\
\text { O sexo feminino } \\
\text { apresentou maior aceitação } \\
\text { aos valores } \\
\text { antropocêntricas. }\end{array}$ \\
\hline 2. & $\begin{array}{l}\text { Nível } \\
\text { de } \\
\text { ensino }\end{array}$ & $\begin{array}{l}\text { V. antropocêntricos } \\
\text { (A) } \\
\text { V. ecocêntricos (E) } \\
\text { V. apáticos (P) } \\
\text { Atitudes (At) } \\
\text { Comportamentos } \\
\text { (Co) }\end{array}$ & $\begin{array}{l}\text { Kruskal Wallis } \\
\text { Anova } \\
\text { Unidirecional }\end{array}$ & $\begin{array}{c}0,46 \\
0,46 \\
\mathbf{0 , 0 2 *} \\
0,68 \\
0,96\end{array}$ & $\begin{array}{l}\text { Significante somente para } \\
\text { valores apáticos. O } 1^{\circ} \text { ano } \\
\text { apresentou maior apatia } \\
\text { ambiental quando } \\
\text { comparado com o } 3^{\circ} \text { ano. }\end{array}$ \\
\hline 3. & Idade & $\begin{array}{l}\text { V. antropocêntricos } \\
\text { (A) } \\
\text { V. ecocêntricos (E) } \\
\text { V. apáticos (P) } \\
\text { Atitudes (At) } \\
\text { Comportamentos } \\
\text { (Co) }\end{array}$ & Kruskal Wallis & $\begin{array}{l}0,51 \\
0,71 \\
0,51 \\
0,74 \\
0,73\end{array}$ & $\begin{array}{l}\text { Nenhum dos resultados foi } \\
\text { considerado significativo } \\
\text { quanto as diferenças com } \\
\text { base na idade. }\end{array}$ \\
\hline
\end{tabular}

Fonte: dados da pesquisa

Quanto ao nível de escolaridade, alunos do $1^{\circ}$ ano apresentaram respostas significativas para maior apatia ambiental quando comparados com o $3^{\circ}$ ano (conforme Tabela 1). VicenteMolina, Fernández-Sáinz e Izagirre-Olaizola (2013) argumentam que a educação formal influencia, claramente, no comportamento ambiental. Diferenças nos resultados com base na idade não foram significativas para nenhuma das variáveis ambientais examinadas.

Foram encontradas correlações entre os valores ecocêntricos (E) e atitudes ambientais (At), valores ecocêntricos (E) e comportamentos ambientais (Co), valores antropocêntricos (A) e apáticos (P), atitudes (At) e comportamento ambientais (Co) com valores-p significante para 0,05, como pode ser visto nas Tabelas 2 e 3 e no modelo estrutural na Figura 3.

Sobre a correlação entre valores antropocêntricos e apáticos a natureza pode indicar que, realmente, os valores antropocêntricos estão mais associados às atividades nocivas ao meio ambiente como discutido em Kopnina (2017) e Kopnina et al. (2018) ao afirmarem que o antropocentrismo tem sido indicado como um dos principais fatores da atual crise ecológica.

Tabela 2 - Resultados do modelo estrutural para os valores ambientais, atitudes e comportamentos ambientais

\begin{tabular}{lcccccc}
\hline \multicolumn{1}{c}{ Relações estruturais } & Hipótese & $\mathrm{f}^{2}$ & $\begin{array}{c}\text { Coeficiente } \\
\text { estrutural }\end{array}$ & $\begin{array}{c}\text { Erro } \\
\text { padrão }\end{array}$ & Valor - P & $\begin{array}{c}\mathrm{R}^{2} \\
\text { ajustado }\end{array}$ \\
\hline $\begin{array}{l}\text { Antropocêntricos -> } \\
\text { Apáticos }\end{array}$ & $\mathrm{H} 1$ & 0,155 & 0,366 & 0,099 & $0,000^{*}$ & 0,128 \\
$\begin{array}{l}\text { Antropocêntricos -> } \\
\text { Atitudes }\end{array}$ & $\mathrm{H} 2$ & 0,015 & 0,125 & 0,122 & 0,305 & \\
$\begin{array}{l}\text { Apáticos } \\
\text { Atitudes }\end{array}$ & & & & & & \\
$\begin{array}{l}\text { Ecocêntricos -> Atitudes } \\
\text { Antropocêntricos -> }\end{array}$ & $\mathrm{H}$ H & 0,013 & $-0,115$ & 0,083 & 0,165 & \\
$\begin{array}{l}\text { Comportamento } \\
\text { Apáticos }\end{array}$ & $\mathrm{H} 5$ & 0,000 & 0,292 & 0,119 & $0,014^{*}$ & 0,097 \\
& & & 0,010 & 0,114 & 0,933 & \\
\end{tabular}


Comportamento

Atitudes ->

Comportamento

Ecocêntricos ->

Comportamento

Antropocêntricos ->

Ecocêntricos

Apáticos ->

Ecocêntricos

$\begin{array}{llllll}\text { H7 } & 0,372 & 0,505 & 0,095 & 0,000^{*} & \\ \text { H8 } & 0,084 & 0,242 & 0,110 & 0.028^{*} & 0,377 \\ \text { H9 } & 0,049 & 0,232 & 0,121 & 0,056 & \\ \text { H10 } & 0,001 & -0,033 & 0,127 & 0,797 & 0,035\end{array}$

Nota: Valores - p estimados por bootstrapping com 5000 repetições e significativos para $0,05 . ; \mathrm{f}^{2}=$ tamanho do efeito de Cohen. H: hipóteses.

Fonte: dados da pesquisa

Tabela 3 - Matriz de correlação entre os valores, atitudes e comportamentos ambientais

\begin{tabular}{lccccc}
\hline & Antropocêntricos & Apáticos & Atitudes & Comportamentos & Ecocêntricos \\
\hline Antropocêntricos & $\mathbf{0 , 5 3 7}$ & & & \\
Apáticos & $0,366^{*}$ & $\mathbf{0 , 6 7 4}$ & & & \\
Atitudes & 0,148 & $-0,054$ & $\mathbf{0 , 8 5 8}$ & & \\
Comportamentos & 0,161 & 0,052 & $0,579 *$ & $\mathbf{0 , 5 2 4}$ & \\
Ecocêntrico & 0,220 & 0,052 & $0,314^{*}$ & $0,406^{*}$ & $\mathbf{0 , 4 9 9}$ \\
\hline Nota: * Valores - p significativo para 0,05 & & & & \\
\hline
\end{tabular}

Fonte: dados da pesquisa

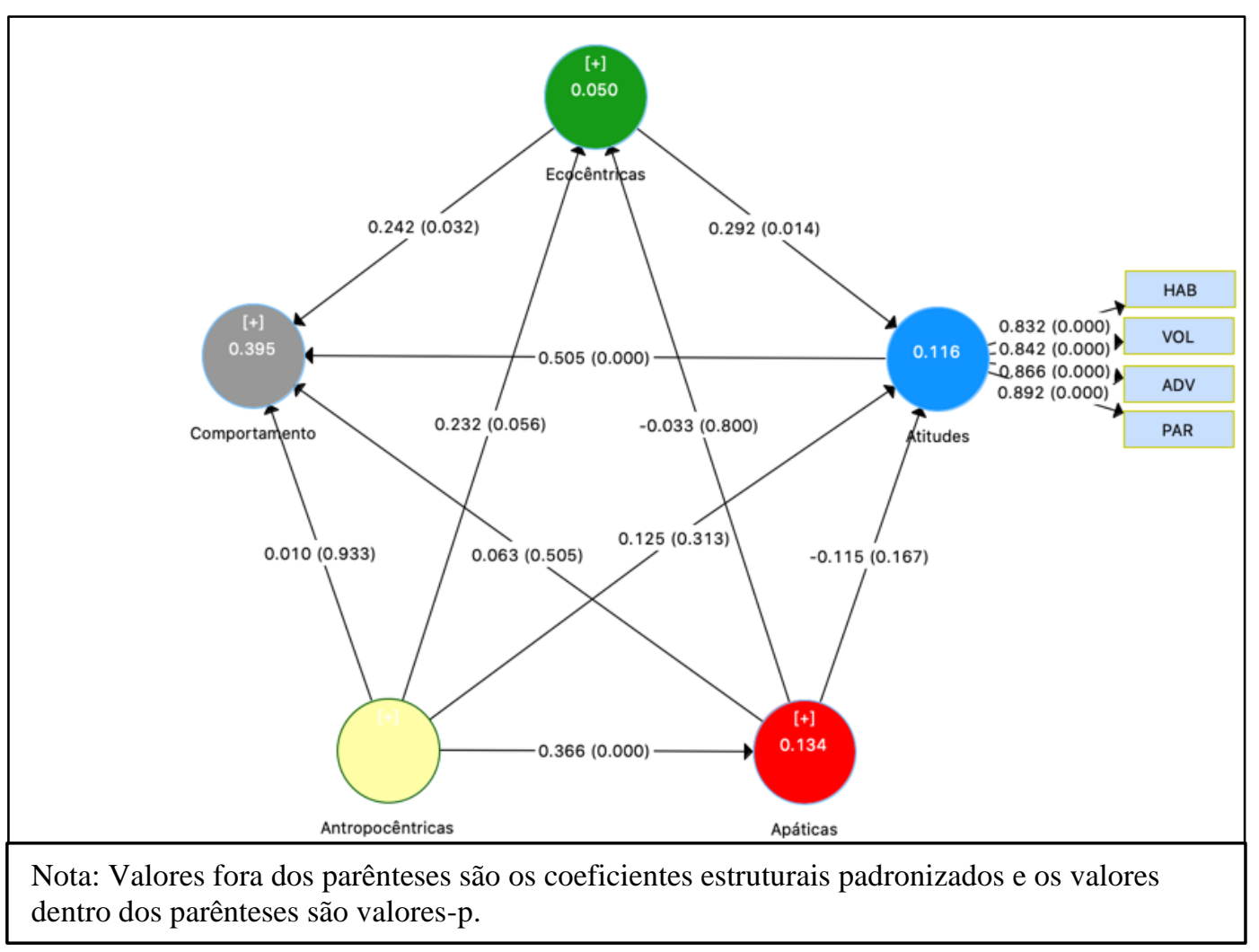

Figura 3 - Modelo estrutural com os resultados do bootstrapping para a correlação entre os valores ambientais, atitudes e comportamentos ambientais

Fonte: dados da pesquisa 
A correlação entre os valores ecocêntricos e as atitudes e com o comportamento ambiental reforça os estudos que indicam que indivíduos com orientação ecocêntrica são mais propensos a agir para proteger o meio ambiente do que os indivíduos com orientações antropocêntricas (THOMPSON; BARTON, 1994; KOPNINA, 2017). Dessa forma, é importante que o planejamento de práticas de Educação Ambiental incentive os valores ecocêntricos em detrimento dos valores antropocêntricos e apáticos, tendo em vista que as hipóteses relacionadas a influência dos valores ecocêntricos sobre as atitudes e comportamentos ambiental foram aceitas.

Heyl, Díaz e Cifuentes (2014) afirmam que a atitude ambiental é um dos fatores que predispõe o comportamento ambiental, e essa afirmação está de acordo com a forte relação encontrada neste estudo entre as atitudes e comportamentos ambientais.

Diante dos resultados, consideramos que nas práticas de Educação Ambiental sejam estimulado o desenvolvimento de valores ecocêntricos, bem como as atitudes ambientais ativas para que, no futuro, reflitam em comportamentos mais adequados ao meio ambiente, que incluem a participação voluntária em sua defesa e em campanhas ambientais.

\section{Conclusão}

Neste estudo, ficou evidente que as escalas de valores (ecocêntricos, antropocêntricos e apáticos), atitudes e comportamentos podem ser aplicados com alto coeficiente de confiabilidade para avaliação diagnóstica e planejamento de práticas ambientais. Além disso, apesar dos valores ambientais apáticos terem sido, de forma geral, menos aceitáveis, é preciso que as práticas de Educação ambiental sejam formuladas a partir da análise de cada um dos itens para que mitos e crenças prejudiciais à relação ambiental sejam desfeitas. Dentre as escalas de atitudes e comportamento, é possível identificar que o voluntariado e a participação direta em atividades de reciclagem e de campanhas ambientais foram os menos aceitáveis e, por isso, devem ser considerados no planejamento de práticas de Educação Ambiental. Diferenças quanto aos valores ambientais, em razão do sexo e da escolaridade, não diferem dos encontrados em outros locais do mundo, e para compreensão desses aspectos os estudos devem ser mais aprofundados, principalmente, com relação às diferenças encontradas entre os sexos para os valores antropocêntricos.

\section{Referências}

ANDRADE, F. M. R. de; GÓMEZ, J. A. C. Educação Ambiental e formação docente na Amazônia brasileira: contextos universitários e realidades cotidianas. Revista Diálogo Educacional, [s.1.], v. 17, n. 55, p. 1598 - 1618, dez. 2017. Disponível em: http://dx.doi.org/10.7213/1981-416x.17.055.ds07. Acesso em: 14 nov. 2020.

AMEMIYA, K.; MACER, D. Environmental Education and Environmental Behaviour in Japanese Students. Eubios Journal of Asian and international bioethics EJAIB, Tsukuba, v. 9, n. 3, p. 109 $115,1999$.

BOCA, G.; SARAÇLI, S. Environmental Education and Student's Perception, for Sustainability. Sustainability, [s.1.], v. 11, n. 6, p. 1553-1571, mar. 2019. Disponível em: http://dx.doi.org/10.3390/su11061553. Acesso em: 1 nov. 2020.

BRASIL. Lei no 9.795, de 27 de abril de 1999. Dispõe sobre a educação ambiental, institui a Política Nacional de Educação Ambiental e da outras providências. Diário Oficial da União, 28 de abr. 1999. Seção 1, p. 1. 
CASALÓ, L.; ESCARIO, J.-J. Heterogeneity in the association between environmental attitudes and pro-environmental behavior: a multilevel regression approach. Journal Of Cleaner Production, [S.L.], v. 175, p. 155-163, fev. 2017. Elsevier BV. http://dx.doi.org/10.1016/j.jclepro.2017.11.237.

CÔRTES, P. L.; DIAS, A. G.; FERNANDES, M. E. da S. T.; PAMPLONA, J. M. V. Environmental Behavior: a comparative study between brazilian and portuguese students. Ambiente \& Sociedade, São Paulo, v. 19, n. 3, p. 113-134, set. 2016. Disponível em: http://dx.doi.org/10.1590/18094422asoc139099v1932016. Acesso em: 1 nov. 2020.

CULVER, L.; MAUCH, C.; RITSON, K. (Orgs.). Rachel Carson's Silent Spring: Encounters and Legacies. Munich: Rcc Perspectives, 2012. 64 p.

DAS, A. K. Development of Environmental Awareness Through the Study of Life-Science in the Secondary Schools of West Bengal. 2013. p. 188. Thesis (Philosophy in Education) - University Calcutta, Calcutta, India, 2001.

DESJARDINS, J. R. Environmental Ethics: An Introduction to Environmental Philosophy. 5 ed. Boston: Wadsworth Publishing Company, 2013.

DIAS, G. F. Os Quinze anos da Educação Ambiental no Brasil: um depoimento. Em aberto, Brasília , v.10, n. 49, p. 3-14, 1991. Disponível em:

<http://emaberto.inep.gov.br/ojs3/index.php/emaberto/article/view/1706>. Acesso em: 14 nov. 2020.

ERTEN, S.; AYDOĞDU, C. The ecocentric anthropocentric and antipathetic attitudes toward environment in Turkish and Azerbaijani students. H. U. Journal of Education, Beytepe, [s.v.], n. 41, inserir n., p. 158-169, 2011.

HEYL, M.; DÍAZ, E. M; CIFUENTES, L. Environmental attitudes and behaviors of college students: a case study conducted at a chilean university. Revista Latinoamericana de Psicologia, Bogotá, v. 45, n. 3, p. 487-500, 2014. Disponível em: http://dx.doi.org/10.14349/rlp.v45i3.1489. Acesso em: 14 nov. 2020 .

KIRKPATRICK, L. A.; FEENEY, B. C. A simple guide to IBM SPSS statistics for versions 20.0 \& 21.0. Belmont, Ca: Wadsworth Publishing Company, 2013.

KOPNINA, H. Evaluating education for sustainable development (ESD): using ecocentric and anthropocentric attitudes toward the sustainable development (eaatsd) scale. Environment, Development And Sustainability, Switzerland, v. 15, n. 3, p. 607-623, out. 2012. Disponível em: http://dx.doi.org/10.1007/s10668-012-9395-z. Acesso em: 14 nov. 2020.

KOPNINA, H. Testing Ecocentric and Anthropocentric Attitudes toward the Sustainable Development (EAATSD) scale with Bachelor students. Rebrae, Curitiba, v. 10, n. 3, p. 457-477, ago. 2017. Disponível em: http://dx.doi.org/10.7213/rebrae.10.003.ao08. Acesso em: 14 nov. 2020.

KOPNINA, H.; WASHINGTON, H.; TAYLOR, B. PICCOLO, J. J. Anthropocentrism: more than just a misunderstood problem. Journal of Agricultural And Environmental Ethics, Switzerland, v. 31, n. 1, p. 109-127, jan. 2018. Disponível em: http://dx.doi.org/10.1007/s10806-018-9711-1. Acesso em: 14 nov. 2020.

KRUSKAL, W. H.; WALLIS, W. A. Use of ranks in one-criterion variance analysis. Journal of the American Statistical Association, Alexandria, v. 47, n. 260, p. 583-621, 1952. 
LANDIS, J. R.; KOCH, G. G. The Measurement of Observer Agreement for Categorical Data. Biometrics, Washington, v. 33, n. 1, p. 159-174, mar. 1977. Disponível em: http://dx.doi.org/10.2307/2529310. Acesso em: 14 nov. 2020.

LIKERT, R. A technique for the measurement of attitudes. Archives of Psychology, New York, v. 140, p. $5-55.1932$.

MACHADO, A. C.; TERÁN, A. F. Educação Ambiental: Desafios e possibilidades no Ensino Fundamental I nas escolas públicas. Educação Ambiental em Ação, Novo Hamburgo, v. 17, n.66, p. 114, 2018. Disponível em: < http://www.revistaea.org/artigo.php?idartigo=3522>. Acesso em: 27 fev. 2020.

MARCATTO, C. Educação Ambiental: Conceitos e Princípios. Belo Horizonte: Feam - Fundação Estadual do Meio Ambiente de Minas Gerais, 2002.

MIHAILOV, N.; SAKELARIEVA, L. Environmental alarmism: the club of Rome and its critics. Studia Ecologiae Et Bioethicae, Warsow, v. 14, n. 4, p. 129-145, dez. 2016. Disponível em: http://dx.doi.org/10.21697/seb.2016.14.4.07. Acesso em: 14 nov. 2020.

MOYANO-DÍAZ, E.; ENCINA, Y.; VICENTE, D. Evaluación del Sistema Nacional de Certificación Ambiental de Establecimientos Educacionales (SNCAE) en Chile: operatoria e impacto. Psicol. Am. Lat., México [s.v.], n.10, p. 00-00, 2007. Disponível em:

$<$ http://pepsic.bvsalud.org/scielo.php?script=sci_arttext\&pid=S1870-

350X2007000200015\&lng=pt\&nrm=iso>. Acesso em: 14 nov. 2020.

MOYANO-DÍAZ, E.; CORNEJO, F.; GALLARDO, I. Creencias y conductas ambientales, liberalismo económico y felicidad. Acta Colombiana de Psicología, Bogotá, v. 14, n. 2, p. 69-77, 2011.

NOVO, F. G. Los primeiros passos de la protección de la Natureza. Homenaje a Rachel Carson. Rev. Acad. Cienc. Exact. Fís. Nat., Madrid, v. 107, n. 1-2, p. 179-194, 2014.

NUNES, M. E. R.; FRANÇA, L. F. PAIVA, L. V. de. Efficacy of Different Strategies in Environmental Education Teaching: association between research and university extension. Ambiente \& Sociedade, São Paulo, v. 20, n. 2, p. 59-76, jun. 2017. Disponível em: http://dx.doi.org/10.1590/1809-4422asoc228r1v2022017. Acesso em: 14 nov. 2020.

PATO, C.; ROS, M.; TAMAYO, A. Creencias y Comportamiento Ecológico: un estudio empírico con estudiantes brasileños. Medio Ambiente y Comportamiento Humano, Tenerife, v. 6 n. 1, p. 5-22, 2005.

PAZ, D. T.; HIGUCHI, M. I. G. Crenças Ambientais Entre Jovens Engajados em Coletivos Socioambientais. Interação em Psicologia, Curitiba, v. 23, n. 1, p. 56-63, abr. 2019. Disponível em: http://dx.doi.org/10.5380/psi.v23i1.55240. Acesso em: 14 nov. 2020.

R CORE TEAM. R: A language and environment for statistical computing. R Foundation for Statistical Computing, Vienna, Austria. 2020. Disponível em: <http://www.R-project.org/>. Acesso em: 27 fev. 2020.

RINGLE, C.; M.; BIDO, D. de S.; SILVA, D. da. Structural Equation Modeling with the SmartPLS. Remark - Revista Brasileira de Marketing, São Paulo, v. 13, n. 2, p. 56-73, set. 2014.

RINGLE, C. M.; WENDE, S.; BECKER, J. M. "SmartPLS 3." Boenningstedt: SmartPLS GmbH. 2015. Disponível em: http://www.smartpls.com. Acesso em: 14 nov. 2020. 
SAKA, M; SURMELI, H. Developing a Scale for Environmental Ethics Approaches. A Study of Validity and Reliability. Journal of Environmental Protection And Ecology, Thessaloniki, v. 3, n. 14, p. 1443-1452, jul./set. 2013.

THOMPSON, S. C. G.; BARTON, M. A. Ecocentric and anthropocentric attitudes toward the environment. Journal of Environmental Psychology, Washington, v. 14, n. 2, p. 149-157, jun. 1994. Disponível em: http://dx.doi.org/10.1016/s0272-4944(05)80168-9. Acesso em: 14 nov. 2020.

VICENTE-MOLINA, M. A.; FERNÁNDEZ-SÁINZ, A.; IZAGIRRE-OLAIZOLA, J. Environmental knowledge and other variables affecting pro-environmental behaviour: comparison of university students from emerging and advanced countries. Journal of Cleaner Production, Amsterdam, v. 61, [s.n.], p. 130-138, dez. 2013. Disponível em: http://dx.doi.org/10.1016/j.jclepro.2013.05.015. Acesso em: 14 nov. 2020.

ANEXO 1: Resultados da frequência absoluto, frequência relativa e moda para cada um dos itens das escalas de valores antropocêntricos, ecocêntricos e apáticos, atitudes, e comportamentos ambientais com os resultados absoluto, relativo e moda.

Legenda: CT: Concordo totalmente; CP: Concordo parcialmente; N: Neutro; DP: Discordo parcialmente; DT: Discordo totalmente; $f_{\mathrm{i}}$ :frequência absoluta; $f_{\mathrm{ri}}$ : frequência relativa; $\mathrm{M}_{\mathrm{o}}$ : Moda. 


\begin{tabular}{|c|c|c|c|c|c|c|c|c|c|c|c|}
\hline \multirow[t]{2}{*}{ Afirmações utilizadas } & \multicolumn{2}{|c|}{$\mathrm{CT}$} & \multicolumn{2}{|c|}{$\mathrm{CP}$} & \multicolumn{2}{|c|}{$\mathrm{N}$} & \multicolumn{2}{|c|}{ DP } & \multicolumn{2}{|c|}{ DT } & \multirow{2}{*}{$\mathrm{M}_{\mathrm{O}}$} \\
\hline & $f_{\mathrm{i}}$ & $f_{\mathrm{ri}}$ & $f_{\mathrm{i}}$ & $f_{\mathrm{ri}}$ & $f_{\mathrm{i}}$ & $f_{\mathrm{ri}}$ & $f_{\mathrm{i}}$ & $f_{\mathrm{ri}}$ & $f_{\mathrm{i}}$ & $f_{\mathrm{ri}}$ & \\
\hline $\begin{array}{l}\text { A1. A pior coisa sobre o } \\
\text { desmatamento das } \\
\text { florestas tropicais é que } \\
\text { ele vai restringir o } \\
\text { desenvolvimento de } \\
\text { novos medicamentos }\end{array}$ & 40 & 0,29 & 50 & 0,36 & 29 & 0,21 & 10 & 0,07 & 9 & 0,07 & 2 \\
\hline $\begin{array}{l}\text { A2. Incomoda-me que os } \\
\text { humanos possam ficar } \\
\text { sem petróleo }\end{array}$ & 28 & 0,20 & 32 & 0,23 & 43 & 0,31 & 21 & 0,15 & 14 & 0,10 & 3 \\
\hline $\begin{array}{l}\text { A3. Ciência e tecnologia } \\
\text { acabarão por resolver } \\
\text { nossos problemas de } \\
\text { poluição, superpopulação } \\
\text { e diminuição de recursos }\end{array}$ & 31 & 0,22 & 46 & 0,33 & 26 & 0,19 & 22 & 0,16 & 13 & 0,09 & 2 \\
\hline $\begin{array}{l}\text { A } 4 . \text { O que mais me } \\
\text { preocupa com o } \\
\text { desmatamento é que não } \\
\text { haverá madeira suficiente } \\
\text { para as gerações futuras }\end{array}$ & 56 & 0,41 & 28 & 0,20 & 20 & 0,14 & 11 & 0,08 & 23 & 0,17 & 1 \\
\hline $\begin{array}{l}\text { A5. Uma das razões mais } \\
\text { importantes para manter } \\
\text { lagos e rios limpos é para } \\
\text { que as pessoas tenham um } \\
\text { lugar para desfrutar }\end{array}$ & 61 & 0,44 & 38 & 0,28 & 13 & 0,09 & 8 & 0,06 & 18 & 0,13 & 1 \\
\hline $\begin{array}{l}\text { A6. Nos precisamos } \\
\text { preservar os recursos para } \\
\text { manter nossa alta } \\
\text { qualidade de vida }\end{array}$ & 102 & 0,74 & 18 & 0,13 & 5 & 0,04 & 3 & 0,02 & 10 & 0,07 & 1 \\
\hline $\begin{array}{l}\text { A7. A mais importante } \\
\text { para a conservação da } \\
\text { natureza é a sobrevivência } \\
\text { humana }\end{array}$ & 57 & 0,41 & 39 & 0,28 & 21 & 0,15 & 11 & 0,08 & 10 & 0,07 & 1 \\
\hline $\begin{array}{l}\text { A8. A natureza é } \\
\text { importante por que pode } \\
\text { contribuir para o prazer e } \\
\text { o bem-estar dos seres } \\
\text { humanos }\end{array}$ & 106 & 0,77 & 22 & 0,16 & 5 & 0,04 & 3 & 0,02 & 2 & 0,01 & 1 \\
\hline $\begin{array}{l}\text { E1. Uma das piores coisas } \\
\text { sobre a superpopulação é } \\
\text { que muitas áreas naturais } \\
\text { estão sendo destruídas } \\
\text { para o desenvolvimento }\end{array}$ & 95 & 0,69 & 27 & 0,20 & 14 & 0,10 & 2 & 0,01 & 0 & 0,00 & 1 \\
\hline $\begin{array}{l}\text { E2. Eu gosto de passar } \\
\text { meu tempo em ambientes } \\
\text { naturais para desfrutar da } \\
\text { natureza }\end{array}$ & 92 & 0,67 & 33 & 0,24 & 10 & 0,07 & 1 & 0,01 & 2 & 0,01 & 1 \\
\hline $\begin{array}{l}\text { E3. Às vezes me } \\
\text { entristece ver florestas } \\
\text { sendo desmatadas para } \\
\text { agricultura }\end{array}$ & 78 & 0,57 & 24 & 0,17 & 26 & 0,19 & 6 & 0,04 & 4 & 0,03 & 1 \\
\hline
\end{tabular}


E4. Prefiro reservas de vida selvagem a zoológicos

E5. Às vezes, quando estou infeliz, encontro conforto na natureza E6. A natureza é valiosa por si mesma

E7. Humanos fazem parte do ecossistema tanto quanto outros animais E8. Uma das razões mais importantes para conservar é preservar áreas selvagens P1. As ameaças ambientais como o desmatamento e a destruição da camada de ozônio são exageradas P2. Parece-me que a maioria dos ambientalistas é pessimista e alguns são paranoicos.

P3. Não acho que o problema do esgotamento dos recursos naturais seja tão ruim quanto muitas pessoas pensam ser P4. Acho difícil ficar muito preocupado com assuntos ambientais P5. Não penso que os seres humanos dependam da natureza para sobreviver

P6. Eu não me importo com problemas ambientais P7. Eu sou contra programas para preservar a natureza selvagem, reduzir a poluição e conservar recursos naturais

P8. É dada muita ênfase para a conservação da natureza

At01.Estou disposto a controlar a utilização da água nas atividades do dia-a-dia (tomar banho, escovar os dentes, lavar roupar) para evitar desperdício At02. Vou verificar e desligar as luzes acessa desnecessariamente

$\begin{array}{cccccccccccc}58 & 0,42 & 31 & 0,22 & 31 & 0,22 & 6 & 0,04 & 12 & 0,09 & 1 \\ 74 & 0,54 & 41 & 0,30 & 14 & 0,10 & 7 & 0,05 & 2 & 0,01 & 1 \\ 122 & 0,88 & 10 & 0,07 & 5 & 0,04 & 1 & 0,01 & 0 & 0,00 & 1 \\ 72 & 0,52 & 39 & 0,28 & 23 & 0,17 & 2 & 0,01 & 2 & 0,01 & 1 \\ & & & & & & & & & & \\ & & & & & & & & & & \\ 81 & 0,59 & 38 & 0,28 & 14 & 0,10 & 3 & 0,02 & 2 & 0,01 & 1 \\ & & & & & & & & & & \\ & & & & & & & & & & \\ 54 & 0,39 & 35 & 0,25 & 17 & 0,12 & 10 & 0,07 & 22 & 0,16 & 1\end{array}$

$$
\begin{array}{lllllllllll}
21 & 0,15 & 33 & 0,24 & 41 & 0,30 & 18 & 0,13 & 25 & 0,18 & 5
\end{array}
$$

$\begin{array}{lllllllllll}12 & 0,09 & 17 & 0,12 & 23 & 0,17 & 22 & 0,16 & 64 & 0,46 & 5\end{array}$

$\begin{array}{lllllllllll}11 & 0,08 & 19 & 0,14 & 21 & 0,15 & 27 & 0,20 & 60 & 0,43 & 5\end{array}$

$\begin{array}{lllllllllll}6 & 0,04 & 12 & 0,09 & 10 & 0,07 & 17 & 0,12 & 93 & 0,67 & 5\end{array}$

$\begin{array}{lllllllllll}2 & 0,01 & 8 & 0,06 & 7 & 0,05 & 23 & 0,17 & 98 & 0,71 & 5\end{array}$

$\begin{array}{lllllllllll}4 & 0,03 & 7 & 0,05 & 10 & 0,07 & 14 & 0,10 & 103 & 0,75 & 1\end{array}$

$\begin{array}{lllllllllll}12 & 0,09 & 22 & 0,16 & 37 & 0,27 & 20 & 0,14 & 47 & 0,34 & 5\end{array}$

$\begin{array}{lllllllllll}109 & 0,79 & 19 & 0,14 & 6 & 0,04 & 1 & 0,01 & 3 & 0,02 & 1\end{array}$

$\begin{array}{lllllllllll}116 & 0,84 & 14 & 0,10 & 6 & 0,04 & 2 & 0,01 & 0 & 0,00 & 1\end{array}$ 
At03. Vou usar a bicicleta ou pegar o ônibus para reduzir a poluição do ar pela emissão de gases por transporte privado At04. Estou disposto a usar papel reciclado porque menos árvores serão cortadas

At05. Estou disposto a reduzir o consumo de produtos desnecessários e embalagens de difícil degradação At06. Vou participar de atividades de plantio de árvores de matas ciliares para proteção dos leitos de rios

At07. Eu serei um membro ativamente envolvido para promoção da utilização de energias renováveis ou limpas At08. Vou tomar medidas contra as empresas e autoridades locais para melhoria do transporte público

At09. Vou participar de projetos a separação dos resíduos e reciclagem At10. Farei campanha para estimula o consumo de produtos orgânicos ou de empresas que possuem responsabilidade ambiental

At11. Vou advertir aqueles que poluem os rios e igarapés

At12. Vou advertir as pessoas sobre o risco do uso de produtos produzidos a partir de fontes não renováveis recursos como petróleo subterrâneo, carvão, gás natural e minas de maneira econômica, em razão da poluição gerada At13. Vou advertir as pessoas sobre a necessidade de optar pelo transporte público para reduzir o consumo de combustíveis fosseis At14. Vou advertir as pessoas sobre a

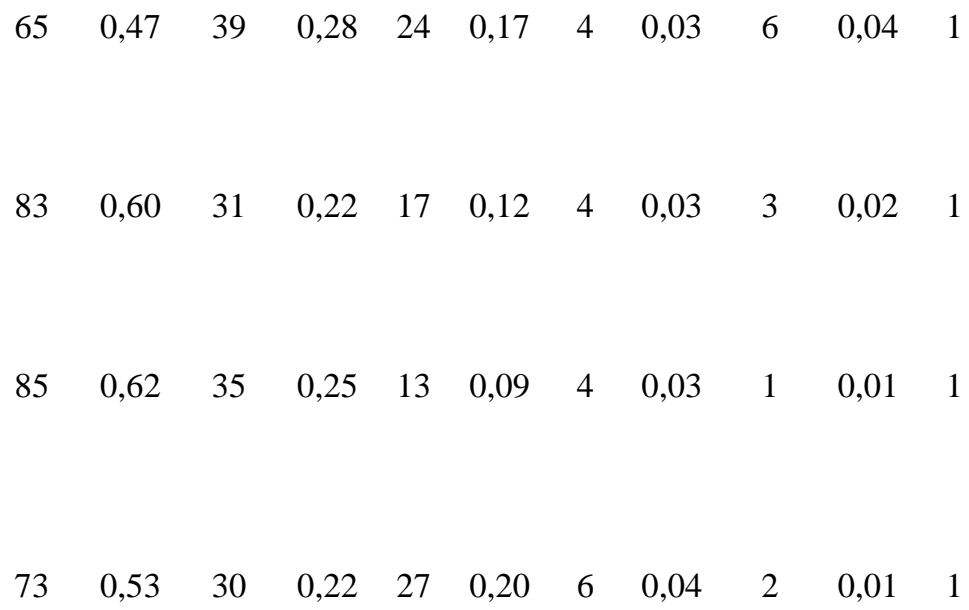


necessidade reduzir, reciclar e reutilizar At15. Vou advertir as pessoas próximas para se abster de consumir $\begin{array}{lllllllllll}80 & 0,58 & 30 & 0,22 & 21 & 0,15 & 2 & 0,01 & 5 & 0,04 & 1\end{array}$ desnecessariamente At16. Eu terei todo cuidado necessário para não polui os rios At17. Tentarei optar pela utilização de energias limpas, como a energia solar, na minha casa

At18. Optarei por transportes que causam o menor impacto a natureza At19. Não prejudicarei a natureza com descarte inapropriado dos resíduos produzidos

At20. Farei o possível para reduzir o impacto na natureza em razão do meu consumo

Co1. Participo de atividades que cuidam do meio ambiente Co02. Eu falo sobre a importância do meio ambiente com os outros Co03. Prefiro consumir bebidas que vêm em garrafas retornáveis Co04. Eu compro produtos orgânicos Co05. Evito usar produtos fabricados por uma empresa que está poluindo o meio ambiente Co06. Eu uso papel reciclado ou certificado Co07. Ando ou uso a bicicleta quando vou a lugares próximos a minha casa

Co08. Zelo pelo

transporte público

Co09. Fecho as torneiras se a água estiver correndo Co10. Economizo água sempre que posso Co11. Desligo a TV quando ninguém está assistindo ou estou fazendo outras coisas Co12. Apago as luzes quando saio de uma sala ou há luz natural $\begin{array}{lllllllllll}119 & 0,86 & 12 & 0,09 & 5 & 0,04 & 0 & 0,00 & 2 & 0,01 & 1\end{array}$ $\begin{array}{lllllllllll}65 & 0,47 & 35 & 0,25 & 34 & 0,25 & 2 & 0,01 & 2 & 0,01 & 1\end{array}$ $\begin{array}{lllllllllll}80 & 0,58 & 34 & 0,25 & 17 & 0,12 & 5 & 0,04 & 2 & 0,01 & 1\end{array}$

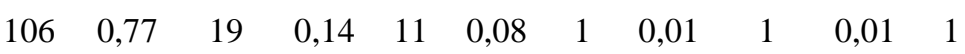
$\begin{array}{lllllllllll}102 & 0,74 & 27 & 0,20 & 7 & 0,05 & 1 & 0,01 & 1 & 0,01 & 2\end{array}$ $\begin{array}{lllllllllll}31 & 0,22 & 34 & 0,25 & 30 & 0,22 & 24 & 0,17 & 19 & 0,14 & 2\end{array}$ $\begin{array}{lllllllllll}71 & 0,51 & 38 & 0,28 & 19 & 0,14 & 4 & 0,03 & 6 & 0,04 & 1\end{array}$ $\begin{array}{lllllllllll}62 & 0,45 & 32 & 0,23 & 32 & 0,23 & 9 & 0,07 & 3 & 0,02 & 1\end{array}$

$\begin{array}{lllllllllll}53 & 0,38 & 34 & 0,25 & 28 & 0,20 & 12 & 0,09 & 11 & 0,08 & 1\end{array}$ $\begin{array}{lllllllllll}38 & 0,28 & 31 & 0,22 & 42 & 0,30 & 12 & 0,09 & 15 & 0,11 & 2\end{array}$ $\begin{array}{lllllllllll}36 & 0,26 & 39 & 0,28 & 36 & 0,26 & 14 & 0,10 & 13 & 0,09 & 1\end{array}$ $\begin{array}{lllllllllll}104 & 0,75 & 19 & 0,14 & 8 & 0,06 & 2 & 0,01 & 5 & 0,04 & 1\end{array}$ $\begin{array}{lllllllllll}90 & 0,65 & 34 & 0,25 & 13 & 0,09 & 0 & 0,00 & 1 & 0,01 & 1\end{array}$ $\begin{array}{lllllllllll}118 & 0,86 & 14 & 0,10 & 5 & 0,04 & 0 & 0,00 & 1 & 0,01 & 1\end{array}$ $\begin{array}{lllllllllll}114 & 0,83 & 20 & 0,14 & 2 & 0,01 & 1 & 0,01 & 1 & 0,01 & 1\end{array}$ $\begin{array}{lllllllllll}117 & 0,85 & 11 & 0,08 & 6 & 0,04 & 3 & 0,02 & 1 & 0,01 & 1\end{array}$ suficiente 
Pesquisa em Educação Ambiental, vol. 16, n. 2, 2021 DOI: http://dx.doi.org/10.18675/2177-580X.2021-15544

Co13. Eu participo de campanhas de reciclagem Co14. Reciclo papel, vidro e latas

Co15. Eu jogo o lixo no local apropriado

\begin{tabular}{rrrrrrrrrrr}
18 & 0,13 & 30 & 0,22 & 40 & 0,29 & 24 & 0,17 & 26 & 0,19 & 3 \\
42 & 0,30 & 39 & 0,28 & 29 & 0,21 & 11 & 0,08 & 17 & 0,12 & 1 \\
107 & 0,78 & 14 & 0,10 & 12 & 0,09 & 0 & 0,00 & 5 & 0,04 & 1 \\
\hline
\end{tabular}

